\title{
Una exploración del impacto de la pandemia en las estrategias digitales de los museos
}

\author{
An screening of the impact of the pandemic on museums' digital \\ strategies
}

\section{Ana Moliner Roca ${ }^{a}$}

${ }^{\text {a Miembro del grupo DIDPATRI (Univeristat de Barcelona, anamolinerroca@gmail.com) }}$

\begin{abstract}
Resumen
¿Estamos asistiendo a una nueva relación de los museos con lo digital?

¿Nos encontramos ante la oportunidad de conectar con el público? ¿Son las nuevas tecnologías una oportunidad para que el museo sea realmente social?

En los últimos 10 años las tecnologías de la información y la comunicación han transformado la didáctica en los centros culturales. A los dispositivos electrónicos 'in situ' se han ido sumando los recursos digitales que el usuario descarga en su propio dispositivo móvil y, sobretodo, las RRSS. Pero no ha sido hasta esta situación excepcional por la pandemia de la sars2-COVID19 en que hemos visto una aceleración en este sentido. Durante los meses de confinamiento (marzo-junio 2020), por las normas de distanciamiento social, los centros culturales permanecieron totalmente cerrados y para poder seguir con su misión divulgadora, seguir manteniendo el vínculo con su público, expandir sus contenidos y, por qué no, atraer nuevas audiencias, optaron por el uso de los recursos digitales.
\end{abstract}

Con el objetivo de diseñar y difundir las futuras propuestas culturales por medios digitales adaptadas a las necesidades de los diferentes sectores de público, diseñamos la Encuesta de Hábitos Culturales en Tiempos de la COVID19 para conocer los hábitos de consumo cultural de la sociedad y el posible impacto que pudiese tener la epidemia de la COVID-19 en los mismos. La encuesta fue lanzada el 18 de abril y cerrada el 21 de junio; participó una variada muestra de 743 personas de todo el territorio español e internacional y pertenecientes a los distintos grupos de edad de los 13 a los 77 años. En este artículo analizaremos los hábitos culturales i la experiencia del usuario en las iniciativas por medios digitales promovidas por los CC durante la pandemia, sus preferencias y necesidades, los canales de difusión que consumen y el grado de motivación a la hora de participar o no en estas iniciativas, todo ello dividido por grupos de edad. 
Las propuestas lanzadas inicialmente por los centros culturales han sido, en su mayoría, la digitalización de los productos presenciales que ya disponía el museo. Los resultados de la encuesta nos dicen que, en general, el público valora estas iniciativas culturales digitales de manera positiva, pero no cree que cambie la idea que la sociedad tiene de los museos. Y es que el público necesita formar parte en la creación de las nuevas propuestas culturales y ven en las tecnologías de la comunicación una oportunidad de participación en el proceso de creación de las nuevas propuestas.

Este trabajo contribuye a entender mejor como la digitalización forzada por la pandemia puede contribuir a una mejor comunicación entre museos y público.

Palabras clave: museo expandido, museo social, digitalización, inclusivo, cocreación.

\begin{abstract}
Are we witnessing a new relationship between museums and digital?

Are we facing the opportunity to engage with the public? Are new technologies an opportunity for the museum to be truly social?

In the last 10 years, information and communication technologies have transformed teaching in cultural institutions. To the electronic devices in situ have been added digital resources that the user downloads on their own mobile device and, above all, the RRSS. But it has not been until this exceptional situation due to the Sars2-COVID19 pandemic that we have seen an acceleration in this regard. During the months of confinement (March-June 2020) by the social distancing regulations, the cultural centres remained totally closed and in order to continue with their dissemination mission, continue to maintain the link with their public, expand their content and, what is more, attract new audiences, opted for the use of digital resources.
\end{abstract}

With the aim of designing and disseminating future cultural proposals by digital means adapted to the needs of different sectors of the public, we designed the Survey of Cultural Habits in the time of COVID19 to know the cultural consumer habits and the possible impact that could have the COVID19 epidemic in them. The survey was launched on April 18th and closed on June 21st. A varied sample of 743 people from all over the Spanish and other international territories ranging from 13 to 77 years participated. In this article we will analyze the cultural habits and user experience in the digital media initiatives promoted by the CC during the pandemic, their preferences and needs, the dissemination channels they consume, and the degree of 
motivation when participating or not in these initiatives, all divided by age groups.

The proposals initially launched by the cultural centres have been, for the most part, the digitization of the face-to-face existing resources. The results of the survey show that, in general, the public values these digital cultural initiatives in a positive way, but does not believe that it will change the idea that society has of museums; since the public needs to play part in the creation of new cultural proposals and believe that communication technologies will be an opportunity for active participation in the process of creating new ones.

This work contributes to a better understanding of how the digitalisation forced by the pandemic can contribute to better communication between museums and the public.

Keywords: expanded museum, social museum, digitalisation, digitization, inclusive, co-creation. 


\section{Introducción}

Con el objetivo de acercar su colección al público, en los últimos años, los museos han implantado las tecnologías de la información y la comunicación entre sus estrategias comunicativas y educativas (Cardona y Feliu, 2013) Podemos dividir estas tecnologías en 2 tipos: las que generalmente se usan en el interior de los muros de la institución (audioguías, códigos QR, Apps) con una finalidad educativa transmisora de información que conviven con la oferta educativa basada en el capital humano (Santacana, 2014) y las estrategias digitales de uso fuera los límites del museo físico como las redes sociales (Facebook, Instagram, Twitter) y otras plataformas (YouTube, blogs, podcast). Las plataformas digitales y las redes sociales formaban parte de la estrategia de comunicación y en contados casos se desarrollaban en ellos acciones educativas y cuando así era su finalidad era atraer a los visitantes a sus centros, donde se desarrollaba la acción. Así, nos encontrábamos ante un escenario donde la fuerte dependencia de la presencialidad por parte de los centros y la oferta culturales en general lo hacía más frágil ante el impacto que iba a recibir (OME, 2020).

La pandemia de la sars-covid19, como un cisne negro (Taleb, 2011) vino de repente i trastocó totalmente el escenario museístico mundial. Hace un año los museos de todo el mundo se vieron obligados a cerrar sus puertas del 14 de marzo al 21 de junio (OPPCC, 2020). La gran mayoría de un día para el otro sin tiempo de reacción ni conocimiento de cuál iba a ser la magnitud de la situación. Lo que en marzo pensábamos que sería una situación crítica, pero acotada temporalmente en un plazo corto, derivó en una cotidianeidad cambiante y compleja. Situación en la que, un año después, todavía nos encontramos y que obliga a los museos a desarrollar su trabajo en unas condiciones en las que la capacidad de adaptación y reacción resulta más necesaria que nunca (OME, 2021).

Bajo la premisa "Lo que no se expone no existe" (Nancy, 2010) los equipamientos culturales, para seguir ejerciendo su misión divulgativa con la sociedad, vivieron una digitalización forzada que puso de manifiesto lo poco preparados que estaban los profesionales de la cultura en las competencias digitales (ICOM, 2020). Estas actividades improvisadas fueron en un inicio la digitalización de las actividades que ya venían realizándose en los centros de manera presencial (OME,2021) sin tener en cuenta las diferencias en el lenguaje, diseño, duración, canales, vías de comunicación y posibilidades que nos pueden bridar los recursos digitales (ICOM, 2020).

Con el paso de las semanas los centros culturales fueron adaptando sus estrategias a estos nuevos lenguajes y, así propusieron iniciativas culturales que demandaban una participación activa del público, así como actividades orientadas a diferentes sectores de edad con la finalidad de conectar con nuevas audiencias (OPPCC, 2020) y conectar así con nuevos sectores de la sociedad.

Justo en este momento de cierre e incertidumbre fue lanzada esta encuesta de públicos desde el grupo de investigación Didpatri para estudiar cómo ha sido la experiencia cultural del usuario en este momento tan excepcional de tres meses de cierre total de los equipamientos culturales y confinamiento domiciliario forzado de la población. Momento en que la cultura 
era más necesaria que nunca (FANCOURT,2019) y los medios digitales una de las pocas vías para su consumo. Ya que con el cierre de los equipamientos culturales la sociedad también sufrió un proceso de digitalización forzada que ha podido cambiar sus hábitos de consumo (WeareSocial, 2021).

\section{Objetivos}

La encuesta fue lanzada con el objetivo principal de conocer el impacto de las normas de distanciamiento social por la pandemia de la SARS-CoV-2 en los hábitos de consumo cultural de la sociedad durante el confinamiento domiciliario y cómo éstos han afectado a las estrategias digitales de los centros culturales.

Para ello diseñamos un cuestionario con preguntas cerradas y de desarrollo (metodología mixta) dividida en 4 apartados: Perfil sociodemográfico, hábitos culturales antes del confinamiento, hábitos culturales durante el confinamiento y perspectivas de futuro.

Todo ello con la finalidad de diseñar las futuras propuestas didácticas de los museos y centros culturales con la participación activa de la sociedad como ésta es la única manera de conseguir llegar a los Objetivos de Desarrollo Sostenible de la Agenda 2030 (ONU, 2015) .

\section{Desarrollo de la innovación}

Lanzada el 18 de abril de 2020 por la IP Ana Moliner, miembro del grupo de investigación DIDPATRI de la Universidad de Barcelona. Se trata del primer estudio de públicos que pretende medir los cambios en los hábitos de consumo cultural durante confinamiento de la población por la pandemia de la COVID-19. Este estudio forma parte de un proyecto de investigación más amplio sobre el uso de las tecnologías de la información y la comunicación en los museos de arte pertenecientes a la Xarxa de Museus d'Art de Catalunya.

Para el diseño de este estudio analizamos las iniciativas que estaban llevando a cabo los diferentes museos y centros culturales las primeras semanas de confinamiento. La metodología de la encuesta es mixta, con preguntas cerradas y de desarrollo y múltiple elección.

Con el objetivo de obtener una muestra de todos los segmentos de la población la encuesta fue diseñada con la herramienta googleforms y fue lanzada en formato digital vía mailig del Grupo de Investigación Didpatri a diferentes profesionales de la cultura invitándoles a que compartiesen para llegar al mayor número de público posible y RRSS (Facebook, Twitter y Instagram) donde se pudo hacer extensiva.

La encuesta estaba dividida en 4 apartados: Perfil sociodemográfico, hábitos culturales antes del confinamiento, hábitos culturales durante el confinamiento y perspectivas de futuro. En el primer apartado, con la intención de definir un perfil sociodemográfico completo, se preguntó por la edad, género, procedencia, estado civil, el grado de estudios, el sector laboral, 
las obligaciones familiares, situación laboral durante el confinamiento, así como la vinculación con el sector cultural. En el segundo apartado, con la intención de conocer los hábitos y prácticas culturales se preguntaba por las actividades que se consumía, la frecuencia y vías por las que se consumía tanto digital como presencialmente, así como el uso que se hacía de las tecnologías. En el tercer apartado, con la intención de conocer los hábitos y prácticas culturales, se preguntaba por las actividades que se consumía, las vías, frecuencia. En el cuarto y último apartado se pedía a los participantes que valoraran los cambios que estaban viviendo en cuanto a sus prácticas culturales y si creían que la situación iba a cambiar las prácticas de la sociedad respecto a la cultura.

\section{Resultados}

Los resultados presentados en esta comunicación son una selección de las respuestas de la encuesta para conocer lo hábitos de consumo cultural tanto presencial como digital antes y durante la pandemia, el consumo y valoración de las diferentes actividades culturales promovidas por los centros culturales y museos durante el confinamiento, los canales digitales por los que se mueven los diferentes sectores de población, así como conocer las necesidades y preferencias de cada sector de la población por grupos de edad (Tabla 1).

Tabla 1. Muestra dividida por grupos de edad

\begin{tabular}{|l|c|c|}
\hline GRUPOS EDAD & MUESTRA & PORCENTAJE \\
\hline Menores de 18 años & 6 & $0,8 \%$ \\
\hline De 18 a 24 años & 86 & $11,57 \%$ \\
\hline De 25 a 34 años & 141 & $18,97 \%$ \\
\hline De 35 a 44 años & 170 & $22,88 \%$ \\
\hline De 45 a 54 años & 157 & $21,13 \%$ \\
\hline De 55 a 64 años & 133 & $17,9 \%$ \\
\hline De 65 a 74 años & 47 & $6,32 \%$ \\
\hline Más de 75 años & 3 & $0,4 \%$ \\
\hline
\end{tabular}

Los 3 meses que la encuesta estuvo abierta (18 de abril al 21 de junio de 2020) participó una muestra total de 743 personas. De estas un $70 \%$ son mujeres frente a un $30 \%$ de hombres. Si dividimos la muestra por procedencia: 690 corresponden al territorio español, de las cuales 396 son de Cataluña y 43 pertenecen al extranjero: 5 de países pertenecientes a la UE 
(Bélgica, España, Francia, Inglaterra y Polonia) y 8 del continente americano (Argentina, Brasil, Chile, Colombia, México, Perú, Uruguay y USA).

El $80 \%$ de las respuestas de la encuesta se obtuvieron durante las 3 primeras semanas en que estuvo abierta (del 18 de abril al 10 de mayo de 2020) Dato que es interesante mencionar ya que los resultados obtenidos corresponden al inicio del confinamiento domiciliario y desconocimiento y desconcierto total de la población.

El cierre de los equipamientos culturales y el confinamiento domiciliario de la población propició un aumento del consumo cultural por vías digitales (Tablas 2 y 3). En la tabla 3 podemos apreciar los cambios de formato de las propuestas culturales y como éstas han producido, en algunos casos, un cambio en los hábitos de consumo de estas actividades del formato presencial al formato digital. A este respecto, cabe lanzar la reflexión sobre la abstención en el consumo de determinados formatos culturales por medios digitales por parte del público. Sin embargo, un 48,6\% de los encuestados reconoce que ha cambiado su manera de consumir cultura y valoran en un 7,2 sobre 10 que esta situación puede afectar a su consumo cultural en el futuro.

En cuanto a los cambios de hábito de participación cultural por medios digitales se aprecia un aumento significativo con un $51,2 \%$ de incremento de media y un crecimiento mayor en el caso del sector más joven de la población (Tabla 2).

Tabla 2. Participación cultural por medios digitales

\begin{tabular}{|l|c|c|c|c|c|}
\hline \multicolumn{2}{c}{$\begin{array}{c}\text { Antes } \\
\text { Confinamiento }\end{array}$} & $\begin{array}{c}\text { Durante } \\
\text { Confinamiento }\end{array}$ & INCREMENTO \\
\hline \multicolumn{1}{|c|}{ Grupos Edad } & Sí & No & Sí & No & \\
\hline Menos de 18 años (6) & $16,7 \%$ & $83,3 \%$ & $100 \%$ & - & $83,3 \%$ \\
\hline De 18 a 24 años (86) & $22 \%$ & $78 \%$ & $91,9 \%$ & $8,1 \%$ & $69,9 \%$ \\
\hline De 25 a 34 años (141) & $36,9 \%$ & $63,1 \%$ & $87,2 \%$ & $12,8 \%$ & $50,3 \%$ \\
\hline De 35 a 44 años (170) & $36,5 \%$ & $63,5 \%$ & $87,6 \%$ & $12,4 \%$ & $51,1 \%$ \\
\hline De 45 a 54 años (157) & $36,3 \%$ & $63,7 \%$ & $85,4 \%$ & $14,6 \%$ & $49,1 \%$ \\
\hline De 55 a 64 años (133) & $39 \%$ & $60,1 \%$ & $85 \%$ & $15 \%$ & $46 \%$ \\
\hline De 65 a 74 años (47) & $37 \%$ & $63 \%$ & $74,5 \%$ & $23,4 \%$ & $37,5 \%$ \\
\hline Más de 75 años (3) & $33,3 \%$ & $66,7 \%$ & $66,7 \%$ & $33,3 \%$ & $33,4 \%$ \\
\hline TOTAL & $35,2 \%$ & $64,8 \%$ & $86,4 \%$ & $13,6 \%$ & $51,2 \%$ \\
\hline
\end{tabular}


Tabla 3. Hábitos culturales del usuario antes, durante (presencial y digital)

\begin{tabular}{|c|c|c|c|c|}
\hline RESPUESTAS & $\begin{array}{l}\text { Antes } \\
\text { COVID19 } \\
\text { Presencial }\end{array}$ & $\begin{array}{l}\text { Antes } \\
\text { COVID19 } \\
\text { Digital }\end{array}$ & $\begin{array}{l}\text { Durante } \\
\text { confinamiento } \\
\text { COVID19 } \\
\text { Digital }\end{array}$ & $\begin{array}{l}\text { INCREMENTO } \\
\text { CONSUMO } \\
\text { DIGITAL }\end{array}$ \\
\hline Ninguna & $0,8 \%$ & $38,2 \%$ & $13,6 \%$ & $+24,6 \%$ \\
\hline Obras de teatro & $55,04 \%$ & $10,9 \%$ & $18,6 \%$ & $+8 \%$ \\
\hline Cine & $82,90 \%$ & $48,3 \%$ & $62,4 \%$ & $+14,1 \%$ \\
\hline $\begin{array}{l}\text { Espectáculos de } \\
\text { danza }\end{array}$ & $20,32 \%$ & $7,1 \%$ & $12,5 \%$ & $+5,4 \%$ \\
\hline Ópera & $13,18 \%$ & $8,3 \%$ & $11,3 \%$ & $+3 \%$ \\
\hline Exposiciones & $75,23 \%$ & - & - & \\
\hline $\begin{array}{l}\text { Exposiciones y/o } \\
\text { visitas virtuales }\end{array}$ & - & $30,6 \%$ & $45 \%$ & $+14,4 \%$ \\
\hline Charlas culturales & $48,18 \%$ & - & - & - \\
\hline $\begin{array}{l}\text { Conferencias } y / 0 \\
\text { ponencias }\end{array}$ & - & $32,8 \%$ & $43,7 \%$ & $+10,9 \%$ \\
\hline $\begin{array}{l}\text { Recreaciones } \\
\text { históricas }\end{array}$ & $12,51 \%$ & - & - & - \\
\hline Documentales & - & $38,4 \%$ & $49,8 \%$ & $+11,4 \%$ \\
\hline $\begin{array}{l}\text { Lecturas poéticas } \\
\text { y/o literarias }\end{array}$ & $20,72 \%$ & $7,9 \%$ & $12,4 \%$ & $+4,5 \%$ \\
\hline $\begin{array}{l}\text { Presentación de } \\
\text { libros }\end{array}$ & $30 \%$ & - & - & - \\
\hline Conciertos & - & $23,1 \%$ & $41,7 \%$ & $+18,6 \%$ \\
\hline $\begin{array}{l}\text { Conciertos en } \\
\text { sales pequeñas }\end{array}$ & $50,74 \%$ & - & - & - \\
\hline $\begin{array}{l}\text { Conciertos con } \\
\text { mucha afluencia } \\
\text { de gente y/o } \\
\text { festivales } \\
\text { música }\end{array}$ & $46,02 \%$ & - & - & - \\
\hline Otros & $6,59 \%$ & $1,3 \%$ & $4,7 \%$ & $+3,4 \%$ \\
\hline
\end{tabular}


Durante el confinamiento, los diferentes museos y centros culturales han dado el salto a la dimensión digital con la digitalización de las propuestas educativas presenciales. Los canales por el que difunden estas iniciativas son los mismos por los que antes anunciaban sus actividades presenciales. Con el objetivo de conocer si estas iniciativas les llegaban al público y el grado de participación en las mismas ofrecemos las dos siguientes tablas donde se puede apreciar los canales de consumo cultural digital del público encuestado y el grado de conocimiento de las actividades (Tablas 5 y 4). Para conocer el grado de motivación y abstención lo hemos calculado el porcentaje de público que conoce las actividades y ha decidido participar en ellas. Así, en general nos da que un 72,1\% de los encuestados conoce estas iniciativas por medios digitales, un porcentaje bajo si recordamos que la encuesta es vía redes sociales y que por lo tanto los participantes en la encuesta suelen ser público de este tipo de canales. Cuanto a la participación y la motivación/abstención vemos como los sectores de edad más avanzada y los de edad más joven son los que cuentan con una menor motivación a la hora de participar. Cuanto a la participación total en estas actividades durante el confinamiento vemos que es de tan solo un $47,2 \%$ de los que están informados, un 35,3\% del total de los encuestados. De este 35,3\% ¿qué tipo de productos consume? (Tabla 6)

Respecto a los canales por los cuales reciben la información de este tipo de iniciativas son por vías digitales y un tercer puesto para el Círculo Social Cercano con la recomendación de amigos y/o familiares se torna importante en este momento de distanciamiento social (Tabla 5). Información dada de manera orientativa por los canales de consumo teniendo en cuenta el sesgo respecto a los canales por los que se ha difundido la encuesta.

Tabla 4. Grado de participación total y participación real

\begin{tabular}{|l|c|c|c|c|c|c|}
\hline \multicolumn{2}{|c}{ Conozco } & \multicolumn{2}{c}{ Participo } & \multicolumn{2}{c|}{ Motivación } \\
\hline Grupos Edad & Sí & No & Sí & No & Participación & Abstención \\
\hline Menos de 18 años (6) & $33 \%$ & $67 \%$ & 0 & $100 \%$ & 0 & $100 \%$ \\
\hline De 18 a 24 años (86) & $66 \%$ & $34 \%$ & $28 \%$ & $72 \%$ & $42 \%$ & $58 \%$ \\
\hline De 25 a 34 años (141) & $70 \%$ & $29 \%$ & $34 \%$ & $66 \%$ & $47 \%$ & $53 \%$ \\
\hline De 35 a 44 años (170) & $76 \%$ & $24 \%$ & $40 \%$ & $60 \%$ & $49 \%$ & $51 \%$ \\
\hline De 45 a 54 años (157) & $67 \%$ & $33 \%$ & $38 \%$ & $62 \%$ & $53 \%$ & $47 \%$ \\
\hline De 55 a 64 años (133) & $77 \%$ & $23 \%$ & $38 \%$ & $62 \%$ & $48 \%$ & $52 \%$ \\
\hline De 65 a 74 años (47) & $75 \%$ & $25 \%$ & $26 \%$ & $74 \%$ & $34 \%$ & $66 \%$ \\
\hline Más de 75 años (3) & $100 \%$ & 0 & $33 \%$ & $67 \%$ & $33 \%$ & $67 \%$ \\
\hline TOTAL & $72 \%$ & $28 \%$ & $35 \%$ & $65 \%$ & $47 \%$ & $53 \%$ \\
\hline
\end{tabular}

Fuente: Moliner, A (2020) 
Tabla 5. Canales de difusión que consumen

\begin{tabular}{|l|c|c|c|c|c|c|c|c|c|}
\hline Canales & $\mathbf{- 1 8}$ & $\mathbf{1 8 - 2 4}$ & $\mathbf{2 5 - 3 4}$ & $\mathbf{3 5}-\mathbf{4 4}$ & $\mathbf{4 5 - 5 4}$ & $\mathbf{5 5 - 6 4}$ & $\mathbf{6 5 - 7 4}$ & $\mathbf{+ 7 5}$ & TOTAL \\
\hline Internet & $83 \%$ & $82 \%$ & $75 \%$ & $76 \%$ & $85 \%$ & $76 \%$ & $81 \%$ & $100 \%$ & $79 \%$ \\
\hline Tv & $100 \%$ & $33 \%$ & $21 \%$ & $22 \%$ & $30 \%$ & $44 \%$ & $34 \%$ & - & $28 \%$ \\
\hline $\begin{array}{l}\text { Redes } \\
\text { Sociales }\end{array}$ & $67 \%$ & $93 \%$ & $90 \%$ & $90 \%$ & $83 \%$ & $84 \%$ & $81 \%$ & $100 \%$ & $87 \%$ \\
\hline $\begin{array}{l}\text { Radio } \\
\text { Círculo }\end{array}$ & - & $13 \%$ & $17 \%$ & $16 \%$ & $18 \%$ & $29 \%$ & $28 \%$ & - & $19 \%$ \\
\hline $\begin{array}{l}\text { Social } \\
\text { Cercano }\end{array}$ & $-33 \%$ & $38 \%$ & $34 \%$ & $35 \%$ & $32 \%$ & $45 \%$ & $33 \%$ & $34 \%$ \\
\hline \begin{tabular}{l} 
Otros \\
\hline
\end{tabular} & - & $4 \%$ & $2 \%$ & $2 \%$ & $4 \%$ & $2 \%$ & - & - & $3 \%$ \\
\hline
\end{tabular}

Tabla 6. Participación en propuestas de los centros culturales por medios digitales

\begin{tabular}{|l|c|c|}
\hline TOTAL & \multicolumn{1}{|c|}{ TOTAL 262/743 } & $81,67 \%$ \\
\hline $\begin{array}{l}\text { Visitas virtuales en museos, } \\
\text { monumentos y yacimientos }\end{array}$ & 214 & $55,34 \%$ \\
\hline $\begin{array}{l}\text { Iniciativas participativas en las } \\
\text { redes sociales }\end{array}$ & 145 & $45,8 \%$ \\
\hline Conferencias i ponencias virtuales & 120 & $50,76 \%$ \\
\hline Descarga de recursos gratuitos & 133 & $22,13 \%$ \\
\hline Talleres y actividades creativas & 58 & $39,69 \%$ \\
\hline $\begin{array}{l}\text { Obras por } \\
\text { especialistas }\end{array}$ & 104 & $4,2 \%$ \\
\hline Otras & 11 & Fuente: Moliner, $A(2020)$ \\
\hline
\end{tabular}

Fuente: Moliner, A (2020)

Para valorar las actividades propuestas por los centros culturales analizamos las iniciativas que estaban lanzando tanto los museos estales como los internacionales en las primeras semanas de confinamiento domiciliario y dividimos estas propuestas en 4 tipos de acciones: formativas, creativas, participativas e interactivas. Las acciones formativas son puramente transmisoras y no esperan una respuesta del usuario más allá de su aprobación y compartición. No espera interacción por parte del usuario. Este tipo de propuestas son las conferencias, ponencias, obras comentadas... realizadas por especialistas. Las acciones creativas son aquellas basadas en un reto creativo y espera una interacción por parte del 
usuario dentro de las premisas de la propuesta. Este tipo de propuestas son los talleres, recreaciones e interpretaciones de obras, etc. Las acciones participativas son aquellas en las que se lanza un reto a los usuarios y éstos participan de manera activa e interactúan entre ellos. Dentro de las propuestas participativas encontramos los retos o 'Challenges' y las que te ponen a prueba sobre determinados conocimientos o 'Quiz'. Por últimos las acciones interactivas son aquellas en que se proponen unas actividades lúdicas al usuario como los juegos o pasatiempos online.

En la tabla 7 podemos ver la valoración de las actividades promovidas por los museos según sus preferencias y a continuación, en la Imagen 1, encontramos una selección de las respuestas cualitativas sobre las preferencias y necesidades del público respecto a nuevas actividades.

Tabla 7. Valoración de las actividades propuestas por los centros culturales

\begin{tabular}{|l|c|c|c|c|c|c|c|c|c|}
\hline $\begin{array}{l}\text { Propuestas } \\
\text { por grupo de } \\
\text { edad }\end{array}$ & $\mathbf{- 1 8}$ & $\mathbf{1 8 - 2 4}$ & $\mathbf{2 5 - 3 4}$ & $\mathbf{3 5 - 4 4}$ & $\mathbf{4 5 - 5 4}$ & $\mathbf{5 5 - 6 4}$ & $\mathbf{6 5 - 7 4}$ & $+\mathbf{7 5}$ & TOTAL \\
\hline $\begin{array}{l}\text { Propuestas } \\
\text { Formativas }\end{array}$ & $16,7 \%$ & $52,3 \%$ & $68 \%$ & $59,4 \%$ & $61,1 \%$ & $66,2 \%$ & $65,9 \%$ & $33,3 \%$ & $61,4 \%$ \\
\hline $\begin{array}{l}\text { Propuestas } \\
\text { Creativas }\end{array}$ & $33,3 \%$ & $43 \%$ & $50,3 \%$ & $48,8 \%$ & $54,1 \%$ & $51,9 \%$ & $44,7 \%$ & $66,7 \%$ & $49,7 \%$ \\
\hline $\begin{array}{l}\text { Propuestas } \\
\text { Participativas }\end{array}$ & $50 \%$ & $38,4 \%$ & $44,7 \%$ & $45,9 \%$ & $47,1 \%$ & $35,3 \%$ & $38,3 \%$ & $66,7 \%$ & $43,4 \%$ \\
\hline $\begin{array}{l}\text { Propuestas } \\
\text { Interactivas }\end{array}$ & $50 \%$ & $48,8 \%$ & $50,3 \%$ & $45,3 \%$ & $44,6 \%$ & $34,6 \%$ & $31,9 \%$ & $66,7 \%$ & $43 \%$ \\
\hline
\end{tabular}

Imagen 1. Preferencias de público para las futuras actividades de los museos 


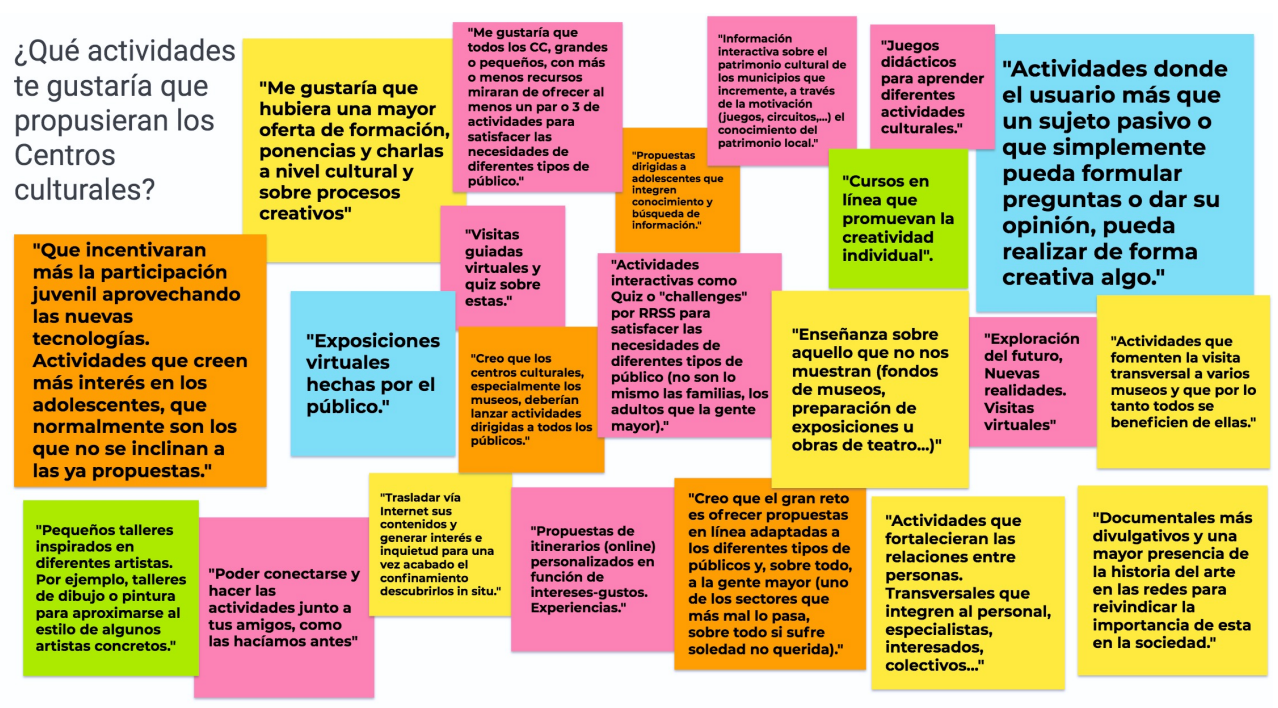

Fuente: Moliner, A (2020)

\section{Conclusiones}

La COVID-19 nos ha colocado en una situación insólita que nos ha hecho cuestionar y cambiar nuestros hábitos y costumbres y recolocar nuestras prioridades y consideraciones más fundamentales (OME, 2021). Desde entonces los museos y centros culturales han ido introduciendo cambios en sus propuestas digitales y sus profesionales han ido adquiriendo competencias digitales con el objetivo de producir una serie de contenidos que acerquen su colección al público. Un público que ahora ha cambiado, ya que desde la aplicación escala global de las normas de distanciamiento social y la consecuente pérdida de público turista las Instituciones se han centrado ahora en su misión social para con el público local (Serra, 2020). En este sentido las estrategias digitales pueden contribuir a conseguir cumplir con los Objetivos de Desarrollo Sostenible de la Agenda 2030 . Fundamentados en la declaración de derechos humanos de la Unesco y en el artículo 27 "Toda persona tiene derecho a tomar parte libremente en la vida cultural de la comunidad, a gozar de las artes y a participar en el progreso científico y en los beneficios que de él resulten" (UNESCO, 2015).

En este sentido, las TIC han sido un excelente medio de acercamiento de los museos hacia su público y quizás una oportunidad de llegar a nuevos públicos. La digitalización hacia un museo expandido que acerque la cultura a la sociedad para conseguir una democratización de la cultura y así un mundo más igualitario y justo (UNESCO, 2015). Pero para ello han de ampliar sus redes, comunicarse por otros canales, proponer nuevas actividades y así poder cumplir su misión social. Las oportunidades que nos brindan las tecnologías de la información y la comunicación son infinitas y van encaminadas a una mayor conexión con el público y la sociedad. Podríamos enunciar las siguientes:

1. Democratizar el acceso a la cultura. Con la digitalización de las iniciativas de los museos se pueden tejer redes entre diferentes partes del mundo y democratizar el acceso a la cultura 
con tan solo un clic. Es cierto que no toda la población mundial tiene el mismo acceso a estas tecnologías, pero este es uno de los compromisos que desde los países del primer mundo deberíamos asumir lo antes posible. Según el estudio realizado por WeareSocial en 2021 en España ha crecido en 8 millones el número de usuarios de redes sociales.

2. Llegar a nuevos públicos usando los canales consumidos por los usuarios de cada segmento de edad y usando el lenguaje para diseñar las propuestas para cada tipo de público (Uffici, 2020). De hecho, vemos como grupos de edad de los sectores más jóvenes han incrementado su consumo y aunque valoran las actividades formativas, prefieren las creativas, participativas e interactivas. Estos grupos de edad son los que menos informados están, en relación con el resto, de las iniciativas llevadas a cabo por los centros culturales estos días y responden a una cuota muy baja de participación. En cambio, un 70,52\% cree que las nuevas tecnologías han facilitado el descubrimiento de muchos museos por un público más amplio y un $52,3 \%$ que atraerán nuevos públicos a sus centros. De ello deducimos que las actividades propuestas en un inicio no les motivan a participar y que los canales por donde se difunden no son los que este segmento de la población consume.

3. Interacción. Permiten hacer un estudio de las prioridades, preferencias y necesidades del público de manera simultánea. La TIC permiten a la institución hacer un estudio de públicos de manera simultánea y precisa al conocer la aceptación del público, la visualización de los contenidos y el índice Rt de compartición de las iniciativas. Además, al permitir una comunicación simultánea con el público y llegar a una parte importante de la población, permite realizar consultas con los usuarios para crear las nuevas propuestas contando con la opinión del público.

4. Democratizar la creación cultural. El público quiere tomar parte en la creación de estas propuestas y las nuevas tecnologías nos pueden ayudar. Las tecnologías han trastocado el sistema tradicional de mercado donde unos especialistas elegían lo que el público iba a consumir y el público podía aceptarlo o no. Las tecnologías y RRSS han brindado una oportunidad, una ventana para que los usuarios se hagan oír, naciendo así la figura del productor-consumidor: prosumer (Toffler, 1980). La inmediatez y la comunicación multidireccional de las TIC pueden ofrecer la cocreación de contenidos y, ahora sí, situar al público en el centro de la acción educativa.

Como vemos, las posibilidades que nos brindan las tecnologías en todos los ámbitos abren una nueva era para experiencia cultural que puede vivida en cualquier parte, por un público más amplio y creada por la sociedad que la consume dando lugar a una nueva concepción de museo abierto a la sociedad; un nuevo museo expandido fuera de lugar, infinito e imaginario, pudiendo trasladarse hasta los no espacios del museo virtual (Solís-Zara, 2016). Y es que las consecuencias de este impacto son un acercamiento de la cultura hacia su misión principal y es que "el museo será social o no será” (Serra, 2020). 


\section{Referencias}

ÁLVAREZ, J. (2021) "Digital report 2021 : el informe sobre las tendencias digitales, redes sociales y mobile" en WE ARE SOCIAL, 27 de enero. <https://wearesocial.com/es/blog/2021/01/digitalreport-2021-el-informe-sobre-las- tendencias-digitales-redes-sociales-y-mobile $>$ [Consulta: 2 de febrero de 2021]

CARDONA, Gemma; FELIU, Maria (2013). "Redes sociales y museos. Cambios en la interacción cultural". Her\&Mus. Heritage \& Museography. $\mathrm{n}^{\circ}$ 13, pp. 83-91. https://raco.cat/index.php/Hermus/article/view/313410/403529 > [Consulta: 10 de abril de 2020]

FANCOURT, D. y SAOIRSE, F. (2019). What is the evidence on the role of the arts in improving health and well-being? A scoping review, Health Evidence Network synthesis report 67, World Health Organisation. Denmark: Copenhagen < https://www.euro.who.int/en/publications/abstracts/what-is-the-evidence-on-the-role-of- the-artsin-improving-health-and-well-being-a-scoping-review-2019> [Consulta: 30 de noviembre de 2020].

GALERIA UFFICI $\quad$ (@uffizigalleries). [Tik $<$ https://www.tiktok.com/@uffizigalleries/video/6925740862894820614?is_copy_url=0\&is _from_webapp $=\mathrm{v} 1 \&$ sender_device $=$ pc\&sender_web_id $=6934786316879463941>$ [Consulta: 20 de febrero 2021]

ICOM (2020). INFORME. Museos, profesionales de los museos y COVID-19: encuesta de seguimiento. $<$ https://icom.museum/es/news/informe-seguimiento-museos-covid-19/> [Consulta: 12 de enero de 2021]

ICOM (2020). INFORME. Museos, profesionales de los museos y COVID-19 $<$ https://icom.museum/wp-content/uploads/2020/05/Informe-museos-y-COVID-19.pdf $>$

[Consulta: 3 de octubre de 2020]

KEMP, S. (2020). "More than half of the people on earth now use social media". WE ARE SOCIAL. $<$ https://wearesocial.com/es/blog/2020/07/digital-2020-july-global-statshot- report> [Consulta: 2 de febrero de 2021]

NANCY, J.L.(2010). Corpus. Ed. Arena Libros, Madrid.

OPPCC (2020) Jornada: escoltar el public presencial, digital i potencial en temps de distància social. $<$ http://observatoripublics.icrpc.cat/cat/activitats/jornada-escoltar-el-public- presencial-digital-ipotencial-en-temps-de-distancia-social.html $>$ [Consulta: 5 de enero de 2021]

OBSERVATORIO MUSEOS DE ESPAÑA. Los museos ante el coronavirus. < http://www.culturaydeporte.gob.es/observatorio-museos-espana/museos-coronavirus.html > [Consulta: 2 de febrero de 2021]

ONU (2015). Objetivos de desarrollo sostenible. https://www.un.org/sustainabledevelopment/es/objetivos-de-desarrollo-sostenible/ [Consulta: 2 de febrero de 2021]

SERRA, P. (2020). Los museos en Cataluña post COVID-19: hacia el museo social. Retos y propuestas de futuro. [Webinar]. Xarxa de Museus d'Art de Catalunya. https://www.youtube.com/watch?v=sDFcptZVNsQ\&feature=youtu.be 
SOLIS-ZARA, S. (2016). El museo vacío. Tesis doctoral. Sevilla: Universidad de Sevilla, < https://dialnet.unirioja.es/servlet/tesis?codigo $=47797>$ [Consulta: 30 de octubre 2020]

TALEB, N.N. (2015). El cisne negro: el impacto de lo altamente improbable. Barcelona: 2011.

TOFFLER, A. (1997). La tercera ola. Barcelona: Plaza \& Janes Editores.

UNESCO. Declaración Universal de Derechos Humanos. $<$ https://es.unesco.org/udhr> [Consulta: 2 de febrero de 2021]

WE ARE SOCIAL. DIGITAL 2021 ESPAÑA. <https://wearesocial.com/es/digital-2021- espana> [Consulta: 2 de febrero de 2021]

\section{Agradecimientos}

Muy agradecida al Dr. Xavier Rubió Campillo, María Feliu Torruella y Sonia Moliner Roca por sus comentarios y ayuda sobre las temáticas tratadas y las versiones preliminares del texto. 